\title{
Zwei Briefe des Berner Professors Johann Georg Tralles an Georg Christoph Lichtenberg (1786)
}

\author{
Von Wolfgang Gresky
}

In der Geschichte der Naturwissenschaften an der Berner Akademie hat man schon früh auf den Einschnitt hingewiesen, der durch den Rücktritt des Professors Niklaus Blauner 1784 und die Berufung von Johann Georg Tralles 1785 gegeben war. Rudolf Wolf hat in seinen Biographien zur Kulturgeschichte der Schweiz Tralles nicht ein eigenes Kapitel gewidmet, weil dieser als Ausländer versäumt hatte, ein Ortsbürgerrecht in der Schweiz zu erwerben; im BlaunerKapitel aber wurde seine Berufung und sein anfangs eifriges und fortschrittliches Wirken ausführlich gewürdigt. Als Friedrich Haag seine Beiträge zur Berner Schul- und Kulturgeschichte schrieb, hat auch er die Zusammenhänge dieser Berufung ausführlich geschildert.

In diesen beiden Büchern kann man nachlesen, was dann in weiteren Darstellungen übernommen wurde, daß man zunächst Professor Sömmering aus Mainz nach Bern holen wollte. daß dieser aber ablehnte, aber auf einen geeigneten Göttinger Fachmann hinwies. Die Bedeutung der jungen Göttinger Universität ${ }^{1}$ für das Schweizer Geistesleben wird auch in diesem Falle deutlich. Der Anatom Sömmering, Freund Forsters, des Schwiegersohnes Heynes, pflegte von Kassel aus rege Beziehungen nach Göttingen und zu Lichtenberg, wie der Schweizer Historiker Johannes Müller, der sich auch um die Besetzung dieser Stelle durch Tralles kümmerte, auch von Göttingen kam. Man holte in Göttingen über Tralles «Testimonia ${ }^{2}$ der zwey gegenwärtig grösten Physikeren und Kenneren der mathematischen Wissenschaften in Europa, des Hofrath Kästners und des Prof. Lichtenbergs » ein, die sehr positiv ausfielen. Lichtenberg urteilte: «Herr Tralles ist einer der besten Köpfe, die mir in den $20 \mathrm{Jahren}$, die ich hier lebe, in diesem Fache vorgekommen sind, seine Hände sind so geschickt als sein Kopf. Bern kann vielleicht einen gleichen finden, aber einen, der mit mehreren Talenten und gröBerem Eifer ganz diesen Wissenschaften lebt, schwerlich.»

Bei dem Schriftwechsel und den Verhandlungen mit Tralles spielte Viktor von Bonstetten eine führende Rolle; auch Johannes Müller setzte sich für ihn ein, indem er dem über Mainz Anreisenden ${ }^{3}$ ein Logis besorgte. Nachdem Tralles wohl am 18.11.1785 in Bern angekommen war, finden wir im Tagebuch des Pfarrers Wyttenbach ${ }^{4}$ einige kleine Notizen über Begegnungen mit ihm. Die Originale der Schulmanuale ${ }^{5}$ des Staatsarchivs Bern halten dann noch ausführlicher alle Einzelheiten der Verhandlungen fest, die schließlich zur Berufung des 
neuen Professors am 14.12.1785 führten. Leider sind im Staatsarchiv nicht mehr die Original-Schreiben «von den gelehrtesten Männern Deutschlands Kästner, Lichtenberg, Heyne, Blumenbach ${ }^{6}$, Büsch und Sömmering » zu finden, von denen Gutachten über Tralles eingingen.

Daß auch der berühmte Altphilologe Chr.G. Heyne ${ }^{7}$ um sein Urteil gebeten wurde, kann wichtig genommen werden, hatte doch Bonstetten erwogen, auch ihn als erste Größe für Deutsch und Latein nach Bern zu holen, ihn bei der Reform des Berner Schulwesens mitwirken zu lassen. Als Heyne seiner Universität an der Leine treu blieb, wirkte er wenigstens auf die Art für Bern, daß er die Berner Studenten in Göttingen bewußt auf ihre künftige Arbeit für den Berner Staat vorbereitete.

Die Ernennung des neuen Professors und seine Arbeitsbedingungen ${ }^{8}$ - Actum coram 200 den 14.12.1785 - sind am besten in dem «Zedel an die Schulräthe» zu fassen, die angewiesen werden, « dem neuerwehlten Hr. Professoren seine Erwehlung kund zu thun, ihme in Ihrem Collegio seinen Rang anzuweisen ». Neben 640 Kronen Gehalt, quartaliter 160 aus der deutschen Standes Cassa zu erheben, ist noch Aufwand für «chymische Experimenta an Acidis und anderen Zuthaten» zur Abrechnung vorgesehen. Bei wöchentlich sechs Stunden Lectiones steht Sitz und Stimme im Oberen und Unteren Schulrat zu; des Rektorats aber ist Tralles enthoben.

Nur bis zu dieser seiner Anstellung wurde im allgemeinen das Wirken von Tralles in Bern in der gedruckten Literatur ausgeführt; es lohnt sich aber, diesen vielversprechenden Neuanfang auch in seinem weiteren Verlauf zu würdigen. Einige seiner Aufgabengebiete seien deshalb hier herausgegriffen:

\section{Visitation der Schulen ${ }^{9}$}

Der Auftrag vom 26.12.1785 lautete, «in den Schulen den Augenschein einzunehmen, wie das Pensum Mathematicum darin betrieben werde und welches die vorzüglichste Lehrart dazu wäre». Am 9.1.1786 betonte der Bericht «die fehlerhafte Lehrart des mathematischen Pensi und rieth zu Einführung Hr. Prof. Klügels Werklein über die Mathematik».

Weil dieses «Werklein» auch noch unzulänglich erschien, beschloß Tralles, selber ein Mathematik-Buch zu schreiben, das dann auch 1788 als Lehrbuch der reinen Mathematik herauskam. Das Exemplar der Göttinger Universitätsbibliothek hat den eigenhändigen Besitzereintrag «Abraham Gotthelf Kästner, Geschenk des Verfassers ». Bei Kästner, der selber eines der vielbenutzten Lehrbücher geschrieben hatte, hatte Tralles Mathematik studiert. Spötter können sagen, daß er von ihm sogar gelernt hat, wie man an der Tafel Fehler macht. Was der Student Gauß ${ }^{10}$ von Kästner überlieferte, berichtet ein Tralles-Schüler ${ }^{11}$ in ganz ähnlicher Art. 


\section{Ergänzung der Bibliothek ${ }^{12}$}

Tralles erhielt am 26.12.1785 den Auftrag, «ein Verzeichnis derjenigen Werke im mathematischen, physischen und chimischen Fach zu verfertigen, welche in hiesiger Bibliothek fehlen und ihr dennoch unentbehrlich sind». Man gewinnt den Eindruck, daß unter Blauner und seinen Vorgängern ein Stillstand eingetreten war, der vor allem in der Sammlung der physikalischen Geräte deutlich hervortrat.

\section{Physikalische Räume ${ }^{13}$}

Der Neuanfang unter Tralles forderte eine neue Lösung der Raumfrage. Der «enge Raum und schlechte Zustand des im alten Spithal zur ExperimentalPhysik bestimmt gewesenen Zimmers» konnte nicht mehr genügen. Wenn zunächst das Sommer-Auditorium zur Verfügung gestellt wurde, ergab sich für Tralles die Notwendigkeit, einen Bedienten zu halten, der die Instrumente aus dem Spital und aus Tralles' Wohnung herbeitrug. Man suchte also nun ein «heiteres wohlgelegenes Auditorium, zudem ein Zimmer zur Aufbewahrung der physischen Instrumente und eine Kammer zu einem chymischen Laboratorio ». Dazu wählte man das Kornhaus an der Ankenwag aus. «Es ist geräumig, heiter, solid und wohl gebaut, hier könnten zugleich noch verschiedene Hörsäle angebracht werden, woran die hiesige Akademie so großen Mangel hat und dadurch die Wissenschaften leiden.»

\section{Das Physikalische Kabinett ${ }^{14}$}

Am 8.12.1785 führte Tralles einem großen Auditorio, in dem aber kaum jemand war, der als Fachmann die Vorführungen hätte beurteilen können, verschiedene Experimente «zur Probe» vor. Da man sah, daß «die Experimente durch die vielfältigen neuen Entdeckungen in der Experimentalphysik, welche durch chimische Versuche zu erweisen sind, jährlich einen ziemlichen Aufwand an Säuren und mehreren Zuthaten erfordern», gestand man Tralles zu, das Notwendige anzufordern und den physikalischen Apparat zu vervollkommnen, zumal die Übergabe der Instrumente am 22.12. durch Professor Blauner Beanstandungen ${ }^{15}$ ergab, daß z.B. «das Scortische Telescopium angeloffen und verschiedene Gläser darin beschädigt seyen». Zugrunde gelegt wurde bei dieser Übergabe ein Verzeichnis aus dem Jahre 1762 !

Eine Überprüfung des Bestandes ${ }^{16}$ durch Tralles und andere Kenner ergab, daß die Instrumente 1750 (vor 36 Jahren also) in Paris angekauft wurden. Der Bericht darüber in der Sitzung vom 23.2.1786 zeigt deutlich, wie veraltet die Sammlung war: «Die Physik war damals noch gleichsam in ihrer Kindheit und 
daher die Instrumente einerseits nur den damahligen Entdeckungen angemessen, andererseits noch roh und ohne Kunst. Hochdenselben ist zur Genüge bekannt, wie beträchtliche und schnelle Progressen diese Wissenschaft seit obigen Zeitpunkt zu ihrer Vollkommenheit gethan hat und wie vielfältige und wichtige Entdeckungen darin gemacht worden; mit dieser Wissenschaft sind zugleich die Hilfsmittel dazu die Instrumente vervielfältigt, verbessert und zum Beweis ihrer Schlüssen tüchtig gemacht worden, verschiedene der ehemaligen massiven und plumpen aber gleichsam unbrauchbar geworden, besonders da viele derselben durch den langen Gebrauch sich abgenuzt befinden.»

Die Fachleute schlugen nun «zum nothwendigen und nützlichen Ankauf ehrerbietigst und unmaßgeblich» folgende Instrumente ${ }^{17}$ vor:

Louisdor

1. Eine gute Luftpumpe mit Exaltations- und Compressionsmaschine für den jezigen Zustand der Physik eingerichtet, mit allem zugehörigen Apparat, dem der in die Lehre von der Luft, in die Hydraulik einschlägt, als auch dem der in Verbindung mit der Elektrizität nothwendig ist. Kostet zusammen bey

2. Eine große Elektrisirmaschine mit vollkommnen Aparat, nebst einer kleinen Handelektrisirmaschine. Dieses zusammen faßt alle die Sachen in sich, die zur Lehre der Elektrizität nothwendig sind

3. Ein magnetischer Apparat, bey

4. Eine Centrifugalmaschine, welche so eingerichtet ist, daß man die Lehre von den Centralkräften vollkommen damit erklären kan, daher in der physischen Astronomie viele Dinge erläutert werden, z. E. Ebbe und FJuth, Keplers Gesetze

5. Eine Hydrostatische Wage mit den zugehörigen Sachen Um den jungen Leuten Begriffe geben zu können, auf welche Art es möglich, daß Weiten des Himmelskörpers voneinander, ihre Größe, Bewegungen so genau haben können bestimmt werden, dienen folgende drey Instrumente, wovon zwey zugleich den Mangel der Fernröhre ersetzen und also die Optik ergänzen.

6. Ein achromatisches Fernrohr mit Objektiv-Mikrometer, welches ungefähr 150 mahl im Durchmesser vergrößert, mit Stativ bey

7. Ein tragbares Observatorium oder Equatoriale beschrieben in la Lande Astronomie

8. Eine Uhr mit einem Stoßpendel bey

9. Ein recht guter Theodolit

10. Zur Optik drey Mikroskopia, nämlich ein zusammen gesetztes, ein einfaches und ein Sonnenmikroskop, so eingerichtet daß es an eine Camera obscura nach der vollkommensten Art, angebracht werden kann. Zusammen bey

12. Ein zusammengesetztes Instrument um die Theorie der einfachen Maschinen zu erläutern und die mechanischen Kräften zu erweisen, bey

13. Zur Erklärung der astronomischen Phänomene der Jahreszeiten, Finsternissen etc. Eine Art hiezu eingerichtete Orrerey, ungefähr 
Wie schnell dieser Antrag bewilligt wurde, finden wir in einem Briefe Lichtenbergs ${ }^{18}$ vom 3.4.1786 bestätigt, in dem der Physikprofessor, der einen großen Hörerkreis hat, beinahe neidvoll nach Bern blickt, weil auf der «Königin der Universitäten » weniger schnell die Wünsche nach besseren Instrumenten erfüllt wurden: «Bern, welches eigentlich nur eine hohe Schule hat, deren Lehrer Professores heißen, hat neulich einem gewissen Tralles, meinem Schüler, den ich dahin gebracht, eine Summe von 500 Carolinen zu den neuesten Instrumenten verwilligt ...»

Noch einen anderen Weg, zu guten Instrumenten zu kommen, hatte aber der Schulrat bereits in der Sitzung vom 9.1.1786 beschritten. Man hatte Nachricht ${ }^{19}$ von "physikalischen Kabinetern in Neus [Nyon] und Lausanne» erhalten, «danach wurde für das dienlichste erachtet den Hr. Prof. Tralles nach Lausanne, Neus und Genf zu senden, um dasige Kabineter und Instrumente in Augenschein zu nehmen, sich des allfälligen Preises zu erkundigen und nachher den Bericht zu erstatten ».

Daß Tralles diese Reise durchgeführt hat, zeigt das Protokoll ${ }^{20}$ der Sitzung vom 9.2.1786: «Erstattete Hr. Prof. Tralles den Rapport über seine Reise ins Pays de Vaud und die Besichtigung der physikalischen Kabineter des Hr. De Lespinasse [Charles de l'Espinasse] in Neus und des Grafen Rasomosski [Grégoire de Razumowsky] in Lausanne». Der Schulrat schlug vor, auf die Sammlung de l'Espinasse zu verzichten. Der Vorschlag, das Kabinett des Grafen Razumowsky anzukaufen, wurde aber so begründet: «Ein nahmhafter Theil des alten physikalischen Aparates allhier ist bey den vielfältigen Progressen in der Physik zu einer größeren Vollkommenheit soviel als unbrauchbar geworden; in anderen Fächern aber, worin man seit einicher Zeit neue Entdeckungen gemacht, herrscht völliger Mangel. In letzterem Fall befindet sich der Aparat der in die Theorie der Luft einschlägt und wovon man hier nicht das geringste besitzt. Wie unentbehrlich ein solcher bey der Experimentalphysik sey, ist jedem Kenner bekannt. Diesem Mangel abzuhelfen bietet sich die beste Gelegenheit dar; Graf Rasomosski welcher sich dermahl in Lausanne aufhält, besitzt einen vollständigen Aparat von Instrumenten in diesem Fach, er bietet solchen um 15 Louisdor zum Kauf an; Hr. Tralles befindet denselben in bestem Zustand und sehr billichen Preis ....»

An dieser Stelle wollen wir mit der Würdigung von Tralles' Wirken in Bern einhalten. Daß er später Grundlagen für die Landesvermessung legte und schon 1786 - auch hier wieder als Schüler Kästners und Lichtenbergs - «Astronomische Beobachtungen " ${ }^{21}$ anzustellen begann, wobei ihm das «obere Kabinet auf dem Kirchhof des großen Münsters» (auf der Münsterplattform) zur Verfügung ge- 
stellt wurde, sei hier ebensowenig untersucht wie die Tatsache, daß später bei ihm negative Charakterzüge hervortraten, durch die ein frühes Aufgeben der Berner Tätigkeit mitbedingt war.

Was bisher mit den Quellen des Berner Staatsarchivs geschildert wurde, die Dienstreise ins Pays de Vaud zur Prüfung anzukaufender Instrumente, das soll nun mit Briefen von Tralles aus jenen Tagen ergänzt und belegt werden.

\section{Zu Tralles' Briefen an Lichtenberg}

An dieser Stelle über Lichtenberg, den großen Physiker der Göttinger Universität, Ausführungen zu machen, erscheint nicht nötig. Die bisher umfangreichste Ausgabe seiner Briefe legte der Hanser-Verlag in Band 4 der Schriften und Briefe vor, die Wolfgang Promies herausgab. Nun plant die Göttinger Akademie der Wissenschaften unter Professor Schöne eine Ausgabe des gesamten LichtenbergBriefwechsels, in dem auch alle Briefe an Lichtenberg, also auch die TrallesBriefe, gedruckt werden sollen. Eben weil diese umfangreiche Ausgabe nicht so schnell zum Abschluß gebracht und vorgelegt werden kann, erschien es angebracht, diese Briefe von Tralles an seinen Lehrer Lichtenberg an dieser Stelle zu veröffentlichen, damit sie der Berner und Schweizer Forschung schon jetzt bekannt werden.

Es sind sechs Briefe, die im Lichtenberg-Nachlaß der Göttinger Universitätsbibliothek erhalten sind (Signatur: Lichtenberg III 229). Die ersten drei stehen mit dem Beginn von Tralles' Wirksamkeit in Bern und seiner Reise vom Januar 1786 in Zusammenhang. Aus Raumgründen konnten hier nur die ersten beiden Briefe vorgelegt werden. Im 4. Brief, von einem ihm «zur Bereicherung seiner physikalischen Kenntnisse» gewährten Urlaub im Juli 1786 über das Berner Oberland geschrieben und bereits indessen veröffentlicht ${ }^{22}$, klingen andere Themen an, wie auch in den Briefen 5 und 6, die vom Plan einer Karte des Kantons Bern oder von Mineralien handeln.

Die Genehmigung zur Veröffentlichung an dieser Stelle wurde dankenswerterweise vom Leiter der Handschriftenabteilung der Göttinger Universitätsbibliothek, Herrn Dr. Haenel, am 2.2.1978 erteilt. Für philologische Beratung und Vergleich der Texte mit den Handschriften habe ich Herrn Ulrich Joost, Mitherausgeber des Lichtenberg-Briefwechsels, zu danken. Die im allgemeinen nicht schwer zu lesende Schrift der Briefe hat eine uneinheitliche Rechtschreibung; die im Plauderton z.T. im Wirtshaus geschriebenen Briefe wenden fast keine Interpunktion an. Am Ende des 1. Briefes entschuldigt Tralles diese Art damit, daß er bei Lichtenberg als Empfänger voraussetzen dürfe, daß er das unzulänglich Ge- 
schriebene erraten könne. Da auch bei den Lesern des Gesnerus dieses Verständnis vorausgesetzt werden kann, wurden buchstabengetreue Rechtschreibung und Zeichensetzung der Handschrift beibehalten. Damit nicht ein umfangreicher Apparat notwendig würde, wurde auf Kennzeichnung der unterstrichenen Stellen, Verbesserungen oder Auswechslung von Worten und einen exakten philologischen Apparat verzichtet; u statt und, od statt oder wurden beibehalten; die Auflösungen von Abkürzungen ${ }^{23}$ wurden z.T. in spitzen Klammern $\langle>$ gegeben.

Merkwürdigerweise berichtet Tralles gar nicht, wie er die «physikalischen Kabinete» besuchte und prüfte; statt dessen wird aus dem Plaudern über den Erlebnisreichtum dieser Tage ein umfangreiches Dokument der Aufklärungszeit. Die Schilderungen der geistigen und Mode-Strömungen wie des Standes naturwissenschaftlicher Arbeiten lassen den Schüler wie ein Spiegelbild Lichtenbergs erscheinen. Selbst bis zur «Gesellschafterin in der Bettlade» spürt man den Einfluß des Meisters, der von «Bettwärmerinnen» spricht. Was hätte es für Lichtenberg bedeutet, wenn er die Einladung in die Schweiz angenommen hätte ? Gerade 1785 waren seine Pläne für eine Italienreise, die ihn auch durch die Schweiz geführt hätte, endgültig aufgegeben. Es gibt ein großartiges Werk ${ }^{24}$ Lichtenberg in England. Wie sähe ein dementsprechendes Werk «Lichtenberg in der Schweiz» dann wohl aus?

Daß ein Ausländer aus dem Norden nicht allen Verhältnissen der Schweiz ganz gerecht wird, ist nicht zu verwundern; daß der Deutsche seine Muttersprache liebte, führte dazu, daß die Briefe zu interessanten Dokumenten deutscher Prosa wurden.

Zwei Briefe von J.G.Tralles an G. Chr. Lichtenberg

Brief 1

Nyon d 14. Jan $86^{25}$

Mein Liebster Freund!

Stellen Sie sich vor! ich habe Ihren äußerst angenehmen Brief heute Morgen d 14 Jan sub Auspiciis augustissimi potentissimi et magnificentissimi Montis Montis Mont-blanc gelesen (denn ich denke daß manches sub auspiciis irgend eines Domini oft geschieht wo er noch nicht mal so viel zu thut als vorgemeldeter) Doch ich will Sie liebster nicht lange mit Phrasen aufhalten, sondern gerade zu das wie geschieht das (des Catechismi) mit Antwort schreiben. Meine gnädigen Herrn (scil Rath zu Bern) haben mir 15 Ld'or zu einer Reise nach Lausanne, Nyon u Geneve gegeben um Instrumente etc zu besehen u den Werth pp zu 
beurteilen u Rapport abzustatten. In Lausanne bin ich gewesen jezt in Nyon u Morgen um 10 hoffe ich in Geneve zu seyn welches nur 2 Meil von hier ist. Hier in Nyon wohne ich beym Hrn Landvoigt von Haller (Sohn des großen) auf ein paar Tage. Wie ich heute Morgen in der Familie beym Thee war, brachte mir der Hr Landvoigt Ihren Brief herein den ich gleich am Siegel erkannte (scil er ist mir von Bern nachgeschickt worden) Die Landvoigtin sagte mir «Den müssen Sie auf der Terrasse lesen» u das that ich denn. Die göttliche Ausicht die ich hier habe können Sie sich gar nicht denken. unten (denn dies Schloß liegt hoch) der Genfer See u die Aussicht auf denselben von Genf an bis Vevey wo die Alpen ein Stück des Horizonts von mehr als $120 \mathrm{Grad}$ einnehmen petit et grand Saleve auch, allein das sind Kinder denn er hat kein graues Haupt im Sommer. Die Dole gukt über den Jura ganz treflich angenehm. Und obgleich es Winter ist so ist doch vieles grün (es versteht sich das keine Bäume blühen) u das Thermometer zeigt +6 Gr. Reaum. Ich mag kein unnüzes Zeug schreiben von der Schönheit u dem Angenehmen, (ich könnte sonst den ganzen Brief damit anfüllen) denn ich bin zu voll $u$ da preßt sich alles so, daß das was ich mir denke oder vielmehr das ensemble dessen was ich beschreiben will ist mir so lebhaft in meiner Imaginazion, daß ichs zusammen niederschreiben will $u$ da kömmt bald dies bald das schöne mir in den Sinn u darüber wird es unordentlich. Ich habe heute viele Stunden gesessen $\mathrm{u}$ gezeichnet $\mathrm{u}$ dann wieder zerrissen. Von Lausanne hieher bin ich immer am Lac de Geneve hergefahren, auch hier so dicht an seinem Ufer auf lauter Cailloux roulé gelaufen, daß mir das Wasser übern Füßen lief. Sie sollten gesehen haben wie froh ich beym Lesen war u wie ich dachte [S. 2] du sollst doch deinen lieben Lichtenberg alles schön schreiben welches du hier siehst u wie angenehm dir zu Sinne gewesen da du seinen Brief gelesen, aber Empfindungen des Herzens lassen sich nicht ausdrücken wie ich jetzt beim Schreiben empfinde - Die Dole gukt jezt durch den Mond erleuchtet wie eine weiße Wolke in meine Fenster herein ich habe ihr aber den Rücken zugekehrt sonst vergesse ichs Schreiben, $u$ guke sie an, $u$ denke Ach wenn doch Sie mein lieber hier bey mir wären wie herzensfroh wir sein würden. Ich bitte Sie um alles wenn Sie mal ein Jahr vergnügt sein wollen so kommen Sie doch zu mir ich will Sie bey mir behandeln wie kein Sohn seinen Vater, mein bestes Zimmer in Bern sollen Sie bewohnen wo Sie die Berner Alpen sehr schön sehen können (u der Anblick auf die Berner Alpen ist schöner wie die Aussicht auf Savoyschen obgleich der Montblanc nicht darunter ist) $\mathbf{u}$ dann wollen wir die Schweiz, Berge $u$ Thäler durchlaufen die Seen überfahren die Berge besteigen die Mädchen besehen pp Wenn Sie hier nicht so gesund $u$ froh werden als ich heute nur immer gewesen bin so glaube ich daß Sie am Paradiese u Adam u Eva nicht glauben. Die Alten 
Commentatoren sind Narrn gewesen daß sie das Paradies absolut in Asien wissen wollen mein Himmel es ist gar kein Zweifel daran, das es der Canton Bern gewesen; ich wills jedem mit 'm Argumentum ad hominem beweisen - nur nicht schriftlich - man muß herkommen. Gefällt's Ihnen dan hier noch länger zu bleiben od Ihr Leben zuzubringen so habe ich den Vorteil davon denn ein Teil Ihres Geistes würde dan doch auf mir ruhen - Ich erinnere einmal zu Woltmann gesagt zu haben ich wünschte (wie in der Bibel ähnlich) daß Kästners Geist zwiefältig auf mir ruhte: er glaubte es sey viel allein ein Jahr später würde ich doch denselben wenigstens dreyfach gewünscht haben - Da wir auf das Verstands Capittel kommen, so muß ich eine Frage thun die Sie nicht schief nehmen müssen. Sie loben mich außerordentlich - Ist das aufrichtig? Sehen Sie mein liebster ich kann recht gut einen Dummkopf von einem verständigen Mann unterscheiden, allein mich selbst kenne ich in diesem Fach gar nicht [S. 3] ich schwöre Ihnen zu daß ich manchen Tag deswegen traurig zubringe weil ich denke mein Gott du bist doch ein Dummkopf. Nicht als ob ich mir selbst nicht bewust wäre das ich in Mathematik etwas weiß u mit der Zeit mehr wissen kann sondern weil ich weiß, daß man ein großer Mathematiker u doch ein Dummkopf seyn kann (exempla sunt impromta ich muß hinzusezen daß ich unter einem gesunden Verstand viel verstehe $\mathbf{u}$ manchen für dumm halte der von der Welt für klug gehalten wird) Ich bin hauptsächlich jezt oft fast zu Thränen auf eine unangenehme Weise gerührt, daß ich denke - Mein Himmel statt deiner könnte die Stelle einen vernünftigeren Mann, der es besser als du verdienet, beglücken der deinethalben jetzt in Sorgen seines Unterhalts stekt $\mathbf{u}$ dadurch an Leib und Seele verdirbt - Doch kein Wort mehr um Sie mein Bester in eine unangenehme Laune zu versetzen - Sie müssen mit einem so schwachen Menschen wie ich bin Geduld haben.

\section{Genéve ce $17^{\mathrm{me}}$ du Mois de Janvier}

Me voila mon cher ami içi il faut que vous me pardonnés de commencer in Französisch denn der Teufel seit 8 Tagen geht fast kein Wort in meiner Muttersprache aus meinem Munde. ich ärgere mich recht daß ich in einem Lande bin (Bern nicht ausgenommen) wo ich statt mich in der kraftvollen deutschen Sprache zu verbessern in Plappern zunehme denn wenn mir auch nur jemand über Tisch eine Platte offerirt so muß ich schon sagen Monsieur, je Vous suis infiniment obligé In Bern verstehe ich das Deutsche nicht $\mathrm{u}$ die Leute die nicht außer Land gewesen sind müssen fremd mit mir sprechen obgleich man mich ziemlich gut versteht. Wahrscheinlich sind Sie Liebster Freund auf Sachen von Genf neugierig Allein diesmal bekommen Sie wenig denn Genf hat meine Laune ganz verderbt 
Der Charakter der Leute hier ist gar nicht mehr deutsch denn der französische Teil des Canton Berns ist es noch u von Genf bis an die Grenze des Canton Berns sind doch keine zwei Stunden zu gehen [S. 4] Am Sonntage den 15 Jan habe ich Bonnet auf seinem Landguth zu Genthod gesprochen u eine Stunde recht vergnügt zugebracht. Er ist ein artiger u verständiger Mann doch ist seine Philosophie noch nicht ganz von Vorurteilen wieder alte hergebrachte Meinungen frey Sie werden merken wovon ich rede) - Sie sagen in Ihrem Brief etwas von ihrer Philosophie bey Gelegenheit eines Buches - ich möchte Ihnen gerne mes Principes philosophiques schreiben allein sie sind nicht gut in die Briefform zu pressen - ich glaube aber wir differiren nicht sehr von einander - (Sie werden wahrscheinlich von meinem Leben in Göttingen mehr wissen als ich Ihnen gesagt habe - Nicht als ob ich mich Ihnen schon damals nicht hätte ganz anvertrauen mögen, sondern weil schweigen ein Charakter der Mannheit ist) $u$ aus dem Handeln erkennt man die Grundsätze eines Mannes besser wie ich denke als aus seinen Schnackereyen. Sie wissen wie ich über das denke was den Pöbel im Zaum hält aber wahrhaftig darauf bin ich stolz daß ich mir bewust bin daß ich nach meinen Grundsätzen doch besser handele als einer der glaubt was alle Gözen nur befehlen können. Allein wie ich mich über so etwas ärgern kann was diese Leute anderen aufbinden kann ich Ihnen gar nicht ausdrücken - wenn ich sehe daß sie's annehmen, denn sonst lache ich dazu. In Bern habe ich Leute gefunden, die an Magnetismus, Physiognomik, Ziehenianismus u des Teufels seine Sachen glauben ich bin 'n paarmal darüber so in Eifer gekommen daß ich mich (u zwar leider in Gesellschaften) ganz vergaß. Allein da die Leute sahen, wie sehr mir die Ausrottung des Aberglaubens am Herzen liegt $u$ ich ihnen sage daß es meine Pflicht ist, so bekehre ich sie (wenigstens einen Teil) wahrscheinlich.

Allein das was Meiners ${ }^{26}$ von Bern gesagt hat (Sömmering war noch bey Tisch) ist nicht wahr. Er mag ein Philosoph sein wie viele [S. 5] Wenn Ziehens Schriften herauskommen sollten, so schicken Sie mir dieselben doch geschwind. Ich habe mir vorgesetzt alle Tollheiten unserer jezigen Zeit vorzunehmen u eine Broschüre zu machen um sie auszupfeifen - denn solche Dinge müssen meiner Meinung nach nicht wiederlegt werden das ist zu viel Ehre. Lavater ist zum Tollhause reif $\mathrm{u}$ zieht doch eine Menge Menschen vom Fürsten bis zum Bettelbuben an sich ich habe verschiedenes von seiner Magnetisation mir erzälen lassen allein für die Authentizität derselben kann ich nicht weiter Bürge seyn als das es seiner Raserey nicht unähnlich sieht. Er hat seine Frau curirt dadurch, daß er ohne sie zu berühren die Luft nach allen Direktionen mit beyden Händen zerteilt hat; sie ist darauf in Schlaf gefallen $\mathrm{u}$ hat schlafend die Medikamente verlangt wodurch sie gesund worden - Wie sich doch die Magnetische Kraft jezt leicht erwecken läßt - ohne 
Stahl Magnet und Eisen - blos der Widerstand der Luft macht daß sie u die Herde weiß was all magnetisch gesund wird. Die Leute in Zürich gehen alle auf den Stelzen des Enthusiasmi, was für Purzelbäume man dadurch sehen wird muß die Zeit lehren. Ich glaube der gesunde Menschenverstand nimmt im Verhältnis des Alters der Welt ab. Ich size zuweilen u denke was ist Wahrheit? Erinnern Sie sich im neuen Testament ${ }^{27}$ wird auf diese Frage (des Pilatus glaube ich) keine Antwort gegeben. Oft habe ich mich in meiner Jugend darüber gequält woher es doch kommen möchte das bei geschikten Leuten die Schriften der Alten in so großem Credit wären - Allein jezt scheint's mir sehr einleuchtend. In den Werken der alten Griechen u Römer findet man bon sens (common sense) bey wie wenig von den Modernen ist dies der Fall? Die Eva hat wahrhaftig nicht vom Baum der Erkentniß Gutes und Böses die Frucht gestohlen sonst wäre die Erbsünde (die Dummheit) nicht in die Welt gekommen doch basta!

[S. 6] Hr Saussure habe ich gesprochen welcher seit 4, Tagen seine Professorstelle niedergelegt hat welche wahrscheinlich Herr Pictet wiederbekommen wird, auch diesen habe ich gesehen er hat hübsche Instrumente. Allein die Leute hier gefallen mir nicht. Sie haben eine suffisance die ins ungeheure geht u machen sich untereinander die abscheulichsten Complimente Senebier habe ich auch gesehen - Er scheint mir ein Mann wie Gmelin nur dessen Bescheidenheit u Höflichkeit fehlt ihm. Er ist ein schmuziger Experimentator sizt in einem Zimmer wo er einige hundert Gläser um sich stehen hat (welche fingerdick mit Staub bedeckt sind) so daß er sich nicht rühren kann u von seiner ganzen Weißheit scheint er nichts gegenwärtig zu haben - denn auch nicht über das mindeste welches die Physik betrift haben wir miteinander gesprochen. Der Vorwurf welchen man ihm macht, daß er seine Versuche gar zu sehr ins kleine macht scheint mir dem Ansehen seiner Gläser pp nach nicht ungegründet zu seyn. Saussure wohnt prächtig im schönsten Hause (Palast könnte man sagen) in Genf er ist durch Heirath reich geworden, von gutem Kopf wie's mir scheint aber von schlechtem Herzen welches ich aus seinem Betragen gegen andere die ihm viel Gefälligkeiten erzeigt haben schließen kann. ich habe ungefähr $3 / 4$ Stunde mit ihm gesprochen Er ließt deutsch u kennt Sie sein Leben wird aber nicht von langer Dauer seyn

Genf ist bey weitem nicht so hübsch als Bern schmuzig u uneben. Allein die Gegend umher ist göttlich angenehm die Schönheit der Gegend um Bern ist anderer Art. Die Häuser sind wie die Leute säuisch in Bern hingegen ist alles nett schön $\mathrm{u}$ rein, die Gegend ist abwechselnd $\mathbf{u}$ veränderlicher obgleich die größere Einförmigkeit der hiesigen keinem lästig wird. Ich kann [S.7] Ihnen gar nicht beschreiben mit welchem unaussprechlichen Vergnügen ich am Sonntag Morgen von 7-12 die Reise am See von Nyon bis hier gemacht habe. obgleich das Wetter 
trüb war so lief das Pferd meiner Meinung nach nie langsam genug um mir alle Freuden die der Anblick der Natur gewähren kann, in jeder Gegend ganz genießen zu lassen, eine ungeheure Menge der schönsten Landhäuser machen die Gegend lebhaft - Bonnet wohnt außerordentlich schön u gewiß ist für einen Philosophen keine bessere Wohnung zu finden als auf dem Lande $u$ in einem Lande wie der Canton Bern. (Den Sommer über werde auch ich auf dem Lande leben) Vielleicht (u zwar ein sehr wahrscheinliches Vielleicht) bringe ichs dahin das ich jedes Jahr 4 Monat frey habe Jul Aug Sept u Okt Allein nächsten Sommer noch nicht da werde ich blos so viel abbrechen um eine Alpenreise ${ }^{28} \mathrm{zu}$ machen - Ich fange deswegen jezt wieder an die Mineralogie hervorzusuchen welche wegen meines schlechten Gedächtnisses außerordentlich verrostet ist - Dabey fällt mir bey kennen Sie schon die französische Uebersetzung der Sciagraphia Regni mineralis von Bergmann? Der Titel ist Manuel du Minéralogiste ou Sciagraphie du regne mineral pp traduite et augmentèe par Mongez. Auteur du journal de physique á Paris 1784 (340 S 80 nebst beynahe $100 \mathrm{~S}$ Introduction) Das Buch bekanntlich ist vortreflich allein auch die Zusäze von Mongez sind sehr interessant $\mathbf{u}$ die Introduktion ist des überlesens wol wehrt, welches sonst gewöhnlich, hauptsächlich bey französischen Schriften sich nicht der Mühe lohnt. Da ich einmal bey Bücheranzeigen bin so will ich zusezen Essai analythique sur l'air pur et les differentes especes d'air par M de la Metherie Doct en Med. á Paris 85.8 ${ }^{0} 474 \mathrm{~S}$. Ich kann jezt (da ich mehr meine 5 Sinne beyeinander haben muß als wirklich in meiner Macht steht) nichts darüber sagen, als daß es neben der Erwehnung der vielen Versuche [S. 8] die über diesen Teil der Naturlehre gemacht sind viel Hypothesen enthält er nennt die dephlogist Luft air u die übrigen fluides aeriformes u hält leztere für bloße Modifikationen der ersteren mit der Feuer oder Lichtmaterie - Doch wahrscheinlich kennen Sies schon u da werden Sie gut thun mirs anzuzeigen das es herausgekommen obgleich ich Ihnen den Titel geschrieben habe.

Ich bedaure recht sehr daß ich in Bern die höhere Mathematik werde auf einige Zeit hintenansezen müssen um über Dinge zu simuliren, blos um sie á la portèe de tout le monde zu machen, allein die Chemie wird wahrscheinlich bey mir dadurch etwas gewinnen. Sie müssens nicht übelnehmen liebster Freund, daß ich so alles durcheinander schreibe $u$ oft nicht interpunctire ich schreibe deswegen mit Vergnügen Briefe für Sie weil ich weiß daß Sie errathen was ich oft schlecht u mit halben Worten ausdrücke, Doppelsinn nicht ausgenommen. Ich schreibe dies hier im Wirthshause $\mathbf{u}$ da ist man überdem nicht so recht wie zu Hause. Morgen reise ich von hier u da der Brief doch nach Bern muß, so nehme ich ihn selbst mit um ihn in Bern noch größer zu machen überdem bin ich hier nicht recht wol auf u gehe nicht zum Abendessen sondern zu Bett. Es hat sich zwar jemand auf be- 
kannte Weise angeboten mein Gesellschafter in der Bettlade zu seyn allein die Miene trägt Zeichen, daß er nicht Neuling in diesem Officio ist $u$ da mag ichs nicht wenn ich auch besser zu Sinne wäre - Ueberhaupt lebe ich recht jezt wies befohlen wird züchtig gerecht $\mathbf{u}$ gottseelig das lezte wissen Sie zu modifiziren $\mathbf{u}$ übrigens hatte ja auch der heil Paulus einen Satansengel der nicht von ihm weichen wollte, $u$ sein dritter Himmel in welchem er entzückt gewesen mag auch wol etwas näher der Erde gewesen seyn als der Sirius -

Gute Nacht Bester Freund!

Brief 2

Bern Jan 86

Gestern bin ich hier wieder so angelangt wie ich weggegangen, nur etwas müder weil ich nicht die Nacht in der Diligence habe schlafen können u dies ist Ursache daß ich gestern nicht am Briefe fortgeschrieben - Vielleicht glauben Sie liebster Freund, daß es schon mit vorigen zwey Bogen genug sey und der Brief so hätte fortgeschickt werden können, allein dass geht nicht «Mit dem Maaß da ihr mit messet wird man euch wieder messen ${ }^{29}$ Sie haben mich mit einem langen Briefe erfreut $\mathrm{u}$ ich suche zu vergelten; wenn Sie nicht so vergnügt bey meinen langen Briefen sind als ich bey den Ihrigen, so liegt die Schuld nicht an meinem Herzen sondern am Kopf, u Sie müssens mit mir machen wie Gott mit den schwachen Menschen u den guten Willen für die That ansehen pp Schreiben Sie mir wenn Sie können immer u oft lange Briefe davon können Sie bey mir wenigstens versichert seyn, daß Sie Ihre Liebe einem Mann von recht gutem Herzen zuwenden Es ist mir eine rechte Freude gewesen daß ich just nur 3 Stunden von Genf war da Sie Haare verlangten, hier folgen drey bey, welche ich vom Paul bekommen u noch mit Mühe car (dit il) je suis grand oeconome avec ces cheveux) - er zog sie doch aus einem Büschel von einigen Hundert allein die Leute in Genf kennen keine Gefälligkeiten. Wenn Sie indessen etwas aus der Schweiz wünschen so sagen Sie's mir nur, denn ich werde um es für Sie zu erhalten gegen die Leute im fordern pp noch unverschämter seyn als ich es je gegen Sie gewesen bin. Paul ist ein exellenter Arbeiter $\mathrm{u}$ bey weitem nicht so schmuzig wie $\mathrm{Kl}$ 〈indworth〉 steht sich auch dem Anschein nach ziemlich gut er ist aber ungeheuer theuer $u$ es ist schwer noch dazu von ihm Sachen zu bekommen. Ich habe verschiedenes bey ihm bestellt - Kennen Sie das Elektrom〈eter > von Saussure ? Ich wills kurz beschreiben es mag ihm seyn wie's will - A ist eine gläserne Glocke von ungefähr $2 \frac{1}{2}$ Zoll hoch a $\mathrm{u}$ b sind Stanniolstreifen (ihrer sind 4 in〈wendig $\rangle$ u 4 auswendig) es hat innen mess〈ingnen〉 Boden wie das Voltaische c ist ein Bogen, dess Mittelp in d, 


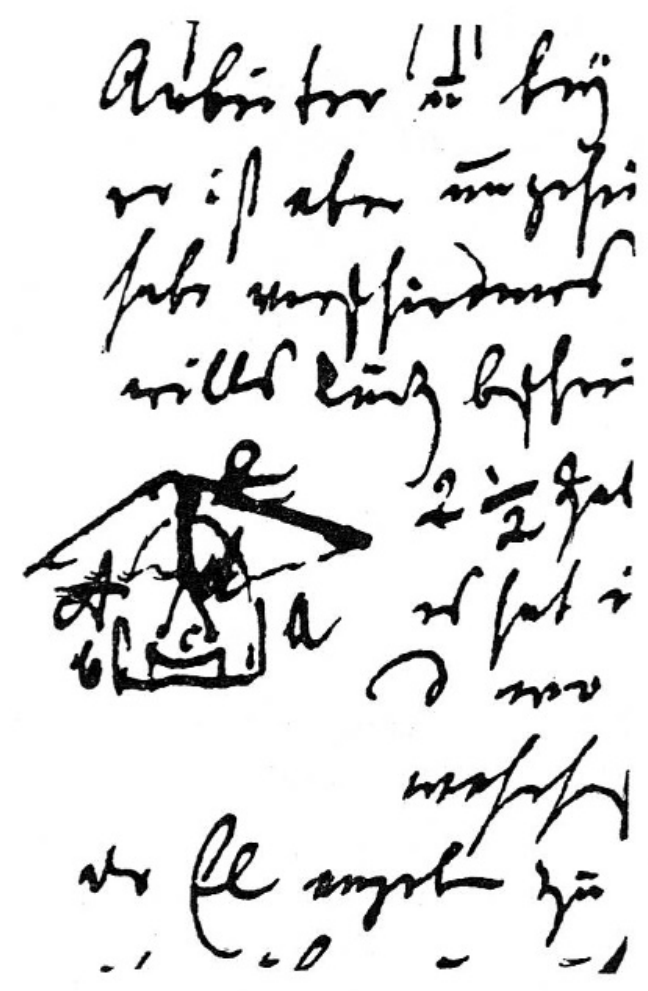

Zeichnung im Brief von Tralles

wo die Faden mit den Kügelchen sich bewegen wie in dem bekannten, welcher (ich habe wahrhaftig vergessen ob in gleiche oder ungleiche Teile) geteilt ist um die Stärke der El angeben zu können auf der Glocke in e kann man einen mess〈ingnen> Schirm schrauben und auf diesen einen Drath der aber sehr spiz - Das Elektrom dient also hauptsächlich, nur die Luftell<ektrizität〉 zu beobachten kostet bey Paul 1 Carl

Ich habe von Gött〈ingen〉 aus einiges $\operatorname{Kupfer}^{30}$ welches ich noch hatte $u$ das sehr unrein war mitgenommen es hat die (von Priestley beob u untersucht) schwarze Materie abgesetzt $u$ ist rein worden, doch war nur sehr wenig von dieser Materie hervorgebracht - Allein ich habe aus dem Cinober erwecktes Quecksilber $^{31}$ gesehen welches von einer Reise aus England hieher gebracht worden $\mathbf{u}$ viel schwarze Materie auch abgesetzt hat $\mathbf{u}$ dadurch unrein träge pp geworden es lohnt sich gewiß der Mühe über diese schwarze Materie mehr Versuche anzustellen als Priestley gethan hat $\mathrm{u}$ in $\mathrm{s}\langle$ einen $\rangle \mathrm{Obs}\langle$ ervations $\rangle$ on different kinds of airs beschrieben ${ }^{32}$

[S. 2] d 22 Jan

Bei Gelegenheit eines englischen Schriftstellers denke ich daran daß ich Sie bitten muß mir durch HE Dieterich die besten englischen Schriftsteller ${ }^{33}$ schicken zu 
lassen denn ich habe sehr wenig in meiner kleinen Büchersammlung z. E. die neu herauskommende schöne Ausgabe von Schakespear, Fieldings Werke, Swift pp ich vergesse sonst mein bischen Englisch u komme dadurch ganz außer der Bekanntschaft mit den Gedanken der hellsten Köpfe. Ueberhaupt ärgert mich das sehr, da ich jezt mich beständig in der neuern Litteratur umsehen sollte, daß ich um das aufzuführende Gebäude anzusehen ich keinen so guten Plaz habe wie olim der liebe Gott wo er den Thurmbau zu Babel betrachtete nur einige von denen die dabey verwirrt worden, kommen mir zu Gesicht.

Eine Neuigkeit - Morgen geht der H Prof Müller ${ }^{34}$ von hier nach Maynz um den höchst traurigen Verlust des hochseeligen Herrn Hofraths Diez zu ersezen, mit $150 \mathrm{Ld}$ Gehalt Titel u was dergleichen mehr ist. Es ist mir recht leid einen vernünftigen Mann in Bern weniger zu sehen u leid einen guten Freund zu verlieren. Wenn man klug genug hier wäre, so könnte man ihn hier behalten od wieder bekommen (welches ich Ihnen im Vertrauen sage) wenn man ihm hier ein Katheder u nur 100 Ld'or gebe - dem armen Mann wird der Abschied von Bern sehr sauer Er sizt immer neben seinem Schnupftuch, welchen er dazu mit Aufrichtigkeit anwendet, wozu ihn die Frauenzimmer gewöhnlich bey sich zu tragen pflegen. Jetzt stehen die Leute hier u sperrens Maul auf über die Sonderbarkeit das Müller weggeht, laufen ihm auf der Straße nach u bitten er möchte doch da bleiben od wiederkommen. Mich verlangt zu sehen ob man sich nicht zulezt wundern wird, daß es doch sonderbar sey daß ein Körper der in die Höhe geworfen ist, dem schadet der sich unter ihn stellt, doch will ich dies nächstens öffentlich demonstriren - Dies hier gefaste bleibt alles unter uns, Sie haben diese Nachricht wahrscheinlich am ersten in Gött<ingen〉 Die ersten 4 Zeilen dieses Absazes können Sie sagen wenn Sie wollen

Sie machen mir eine außerordentliche Freude, wenn Sie mir eine Copie von unsers Hrn Jesu Christi (alias Newtons) Bild ${ }^{35}$ ohne ihren Schaden schicken können. Es ist hier ein sehr geschickter Künstler der Büsten aus Erde ${ }^{36}$ (und anderen Sachen) ganz vortreflich macht, der soll mir wieder eines nach dem machen, welches ich über den physischen Altar im [S. 3] neuen Hörsal welcher für mich gebaut werden soll fixiren lassen will, was Sie schicken will ich bey mir behalten

Die Lampe u Micros comp werden mir willkommen seyn, wollen Sie nur zusehen so guten Kauf als möglich bey beyden zu machen. ich sehe daß ich hier gut darauf achten muß schöne Sachen zu haben u hübsche Experim zu machen, sonst verzweifeln die Leute an der Physik ich habe vor mir selbst ein Cabinet de physique experiment zu machen um Meister meiner eigenen Versuche zu seyn. Fürs erste habe ich mir vorgenommen die geographische Lage von Bern Deklinat $u$ Inklinat der Magnetnadel zu bestimmen um letztere beyde genau zu bekommen 
habe ich eine besondere Idee. Wenn Sie glauben daß dies ein Artikel für Ihr Magazin $^{37}$ seyn könnte so will ich Ihnen wenns vollendet, kurz abgefasst schicken ich habe noch verschiedene andere Versuche in petto allein ich muß warten bis die Instrumente dazu vollendet sind.

Für gute Lehren, Bester Freund machen Sie in Zukunft keine Vorreden wie können Sie glauben daß ich Sie scheel ansähe und um Ihnen einen guten Rath zu geben, so wiederhohle ich meine Bitte kommen Sie zu mir, Sie leben alsdann gewiß wenigstens (Ihre Lebenszeit heisse $x$ Jahr) $x+x^{\frac{1}{2}}+x^{\frac{1}{3}}$ et sic in infinitum, $\mathrm{u}$ wir wollen fröhlich $\mathrm{u}$ gutes Muths seyn $\mathrm{u}$ zusammen experimentiren $\mathbf{u}$ philosophiren bey erstern kann ich Ihnen doch wenigstens an die Hand gehen; an hübschen Instrumenten solls nicht fehlen. Was haben Sie jezt davon in Gött<ingen> zu leben, Sie sind da doch zu geplagt, Sie können nirgends freyer seyn als hier wenn Sie Leute sehen wollen so bekommen Sie so viele Bekanntschaften als Sie wollen, wollen Sie das nicht so lassen Sie die Leute ihrer Wege gehen. Der HE Rathsherr v Tillier ${ }^{38}$ u s Frau habn mir viele Complimente an Sie aufgetragen u sagen sie halten sich sehr verbunden für alle die Güte welche Sie ihrem Sohn erzeigen - ich schreibe dies kalt hin, kann Ihnen aber nicht schreiben mit welcher Herzenswärme mir diese Grüße aufgetragen worden. - Sie machen meinen Freunden u Bekannten auch den Bernern ${ }^{39}$ meine Komplimente u sezen so viel dazu u sagen so viel als Sie für gut halten denn ich kann unmöglich an alle schreiben.

[S. 4] Noch einige Bitten. Können Sie mir einen großen Cylinder zu einer Elektrisirmaschine verschaffen (der gefaßt wäre, ich möchte gerne einen englischen haben) allein so groß als möglich um die großen Experimente machen zu können. Ferner ein Canisches ${ }^{40}$ Elektrometer $u$ einen allgemeinen Entlader. Wenn Sie liebster Freund mir irgend ein hübschgemachtes u gutes Instrument schicken können (außer es möchte ungeheuer theuer seyn) so kömmt es mir gelegen, denn diese müssen mich hier Lüstre geben $\mathbf{u}$ ich will für Ihre Väterliche Vorsorge für mich so erkenntlich seyn als es in meinen Kräften ist. Sie haben ohne Zweifel schon von der in England neu erfundenen Luftpumpe ${ }^{41}$ gehört. Auch dies Instrument möchte ich mir gerne anschaffen allein so vollkommen als möglich - Eine Parkersche Maschine käme mir auch gelegen denn Glas Sachen kann ich hier gar nicht bekommen. ich hatte kürzlich Glocken bestellt u bekam Bouteillen mit 2 Hälse.

Mein liebster so bald als möglich antworten Sie doch, die Zeit die Sie zu meinen Briefen verwenden soll gewiß nicht ganz verlohren seyn - denn wenigstens gehen doch 20 Tage hin ehe ich wieder Antwort haben kann, also ungefähr im Jahr so viel Briefe als Mondjahre (das Mondjahr zum tropischen Mondumlauf um die 
Erde gerechnet) hätten Sie zu schreiben u auch ich, wenn ich Materie habe, so schreibe ich gern mehr u wenn Sie mehr schreiben können, so sind dies meine schönsten Geschenke aus Deutschland - Sagen Sie mir doch auf welche Art ich am besten Geld nach Gött<ingen〉schicken kann grade zu durch Briefe ? od sonst. Leben Sie recht vergnügt $\mathrm{u}$ wohl

NB dr Brief geht heute Sonntag ${ }^{42}$ d 22 Jan ab.

Ihr Ihnen ganz ergebener

$\left\langle\right.$ Notiz Lichtenbergs : > erhalten Dienstag ${ }^{43} \mathrm{~d} 31^{\text {ten }}$

Tralles

\section{Personen-Register}

Es erschien angebracht, die Lebensdaten der in den Briefen genannten Personen statt in den Anmerkungen in einem gesonderten Register zu verzeichnen. Fast alle Naturwissenschaftler und Gerätebauer, mit denen Tralles zu tun hatte, begegnen auch in Lichtenbergs Briefen. Aus dem Register zu diesen konnten deshalb weitgehend die Daten übernommen werden.

Bergman, Torbern, 1735-1784, Professor der Chemie in Upsala

Blauner, Niklaus, 1713-1791, seit 1752 Professor in Bern, vorher Studienaufenthalt in Paris und Göttingen, 1784, Resignation

Bonnet, Charles, 1720-1793, Naturforscher und Philosoph, korrespondierendes Mitglied der Göttinger Sozietät

Bonstetten, Karl Viktor von, 1745-1832, Wahl in Schulrat 1781, Landvogt in Nyon 1787 bis 1793

Büsch, Johann Georg, 1728-1800, Direktor der Hamburger Handelsakademie, Förderer von Tralles; 1773 Lichtenberg besucht ihn

Canini, Giuseppe Maria Simone, 1720-1796, Abbate in Venedig, Gerätebau, Magnete, Kompasse

Dieterich, Johann Christian, 1722-1800, Lichtenbergs Verleger, Hauswirt und Freund, Herausgeber des Göttinger Magazins

Dieze, Johann Andreas, 1729-1785, neben Heyne 2. Bibliothekar in Göttingen, seit 1784. in Mainz

Gmelin, Johann Friedrich, 1748-1804, 1775 Prof. der Medizin in Göttingen, 1782 Begründer des Chemischen Institutes

Haller, Gottlieb Emanuel von, 1735-1786, Historiker, ab 1784 Landvogt in Nyon, stirbt auf Dienstreise nach Bern kurz nach Tralles' Besuch Ostern 1786; «nützlicher Korrespondent» der Göttinger Akademie. Seine Frau war Anna Margaretha geb. Schultheß aus Zürich.

Heyne, Christian Gottlob, 1729-1812, führender Kopf der Göttinger Universität (Bibliothek, Akademie), Archäologe, Betreuer der Schweizer Studenten der Universität

Hurter, Johann Heinrich, 1734-1799, von Schaffhausen, königlich-britischer Hofmaler, Fabrik physikalischer Instrumente in England

Kästner, Abraham Gotthelf, 1719-1800, seit 1756 Prof. der Mathematik und Physik in Göttingen, auch astronomische Arbeit 
Klindworth, Johann Andreas, 1742-1813, Uhrmacher, Mechanikus in Göttingen, arbeitet für Lichtenberg, ab 1784 Hofmechanikus in Gotha

La Métherie, Jean-Claude de, 1743-1817, französicher Naturforscher (nicht zu verwechseln mit La Mettrie)

Lavater, Johann Caspar, 1741-1801, religiöser Schriftsteller in Zürich, Physiognomische Fragmente von 1775-1778, von Lichtenberg angegriffen, im Juni 1786 zu Besuch bei Lichtenberg, der guten Eindruck gewinnt, "von Magnetismus nicht gesprochen »

Lichtenberg, Georg Christoph, 1742-1799, Physikprofessor in Göttingen, berühmte Versuche, vielseitiger Naturwissenschaftler. Lehrer von Tralles

Lichtenberg, Ludwig Christian, 1738-1812, Bruder des vorigen, Legationsrat in Gotha, gab heraus: Magazin für das Neueste aus der Physik...

Mongez, Jean-André, 1751-1788, Herausgeber des Journal de physique 1779-1785, dann Begleiter der Weltumsegelung von La Pérouse

Müller, Johannes von, 1752-1809, Historiker der Schweiz, Göttinger Beziehungen zu Heyne, Forster, Lichtenberg, 1807/08 Kurator der Universität, förderte Tralles

Newton, Isaac, 1643-1727, Physiker, Astronom, angebeteter Meister von Lichtenberg, Tralles, später von Gauß

Paul, Mechaniker in Genf, arbeitete für de Saussure; 1784 erhielt Lichtenberg Haarhygrometer von ihm, 1788 ein Thermometer

Pictet, Marc-Auguste, 1752-1825, seit 1786 Prof. in Genf, später Präsident der physikalischen Gesellschaft Genfs

Priestley, Joseph, 1733-1804, englischer Naturforscher; 1774 Sauerstoff entdeckt; 1775 Lichtenberg besuchte ihn in London

Saussure, Horace-Bénédict de, 1740-1799, Prof. in Genf; 1783 Haarhygrometer erfunden; 1786 Lichtenberg liest seine "Reisen in die Alpen»

Senebier, Jean, 1742-1809, ursprünglich Pfarrer, dann Bibliothekar in Genf, Physiker, Chemiker und hervorragender Pflanzenphysiologe

Short, James, 1710-1768, Mechanikus in London, bedeutende Spiegelteleskope

Sömmering, Samuel Thomas, 1755-1830, Anatom in Kassel, von da Besuche in Göttingen bei Lichtenberg, seit 1784 in Mainz, später in München und Frankfurt a. M.

Woltman, Reinhard, 1757-1837, Wasserbaukondukteur in Ritzebüttel, Erfinder des Wassermeßflügels; Anregungen für Lichtenbergs Seebäder-Pläne

Ziehen, Konrad Sigmund, 1727-1780, Superintendent in Zellerfeld, weissagte Erdrevolutionen, wogegen Lichtenberg zwei Aufsätze schrieb.

\section{Anmerkungen}

1 Die 1737 feierlich eingeweihte Landesuniversität des Königreichs Hannover, die weitgehend den Naturwissenschaften aufgeschlossen war, wurde von vielen Schweizer Studenten besucht.

${ }^{2}$ Haag, Friedrich, Beiträge zur Bernischen Schul- und Kulturgeschichte, 1. Band, 2. Hälfte, Bern 1900, S.122.

3 Tralles schrieb am 11.11.1785 aus Mainz einen Brief an J. Müller; s. Haag (Anm. 2), S. 300 . 
4 Haag (Anm. 2), S.129.

5 Schulrat Manuale 14 (1783-88) = Staatsarchiv Bern B III 885, S.105-152, etwa 35 wichtige Stellen, daneben B III 886 = Schulmanuale 15 und Polizeibuch 18, S. 164, und Ratsmanual 381, S. 289 und 419.

6 Manual 14 (s. Anm. 5), S. 118, hier Hayne und Blumbach geschrieben.

7 Haag (Anm. 2), S. 465.

8 Manual 14 (Anm. 5), S.119 f.

9 Ebenda S. 125 und 133.

10 Student Gauß zeichnete Kästner, der an der Tafel falsch rechnete; s. Gauß-Gesellschaft Mitteilungen Nr. 10, S. 3.

11 Wolf, Rudolf, Biographien zur Kulturgeschichte der Schweiz, Band 1, Zürich 1858, S. 334-340, hier S. 337.

12 Manual 14 (Anm. 5), S. 125.

13 Ebenda S. 126, 129, 139.

14 Ebenda S. 119 und Wolf (Anm.11), S. 336.

15 Manual 14 (Anm. 5), S.124.

16 Ebenda S. 147.

17 Ebenda S.149.

18 Lichtenberg-Briefe (Hanser-Ausgabe Band 4), S. 663.

19 Manuale (Anm. 5), S.132.

20 Ebenda S. 140.

21 Ebenda S.151.

${ }^{22}$ Gresky, Wolfgang, Ein Brief von 1786 über das Berner Oberland, Tralles an Lichtenberg, Historisches Museum Schlo $\beta$ Thun, Jahresbericht 1973.

${ }_{23}$ Nur wenige Abkürzungen wurden aufgelöst, weil Fachliches vorausgesetzt werden kann.

${ }^{24}$ Lichtenberg in England, Dokumente einer Begegnung, hrsg. von H. L. Gumbert, Wiesbaden 1977, 2 Bände.

25 In der Handschrift versehentlich «85».

${ }^{26}$ Meiners, Christoph (1747-1810), seit 1775 Professor in Göttingen, schrieb Briefe über die Schweiz, Band 1-4, Berlin 1784-1790. Die Reise fand 1782 statt; über Bern Band II.

27 Johannes 18, 38.

${ }^{28}$ Alpenreise = Brief 4, s. Anm. 22.

29 Biblisch = Matth. 7, 2 .

${ }^{30}$ In Handschrift astronomisches Zeichen für Venus = Kupfer.

${ }^{31}$ In Handschrift astronomisches Zeichen für Merkur = Quecksilber.

32 Auf der Göttinger Universitätsbibliothek sind fast alle hier in den Briefen genannten Werke vorhanden, so daß leicht genaue bibliographische Angaben zu überprüfen wären.

${ }^{33}$ Bei Dieterich in Göttingen waren 1784 2 Bände erschienen: Works of the English Poets with Prefaces biographical and critical, besprochen Göttingische gelehrte Anzeigen 1785 I, S. 241.

34 Alle Daten über Müllers Abschied s. Karl Henking, Johannes von Müller, Band II, Stuttgart 1928, S. $83 \mathrm{ff}$.

${ }^{35}$ Newton, wie Jesus am 25.12. geboren; Lichtenbergs ausführliche Schilderung der Totenmaske, s. Briefe (Hanser Band 4), S. 682 und 713. 
36 Terracotta; Anmerkung des Verfassers unter dem Text: «Diese Erde wird nachher gebrannt und dadurch so hart, daß sie mit dem Stahl Funken gibt.»

37 Die Ausgaben des Magazins und ihr Inhalt sind erfaßt bei: Lauchert, Friedrich, G. Chr. Lichtenbergs schriftstellerische Thätigkeit, Göttingen 1893.

${ }^{38}$ Ratsherr v. Tillier, z. B. am 19.12.1785 in der Anwesenheitsliste im Schulrat neben Tralles genannt (Manuale S.119), hatte einen in Göttingen studierenden Sohn, dem Lichtenberg am 20.4.1786 ein Empfehlungsschreiben nach Berlin mitgibt (Briefe S. 666).

39 «Die Berner» Studenten in Göttingen waren eine feste Gemeinschaft, wie sie sich nach ihrer Rückkehr als «die Göttinger» in Bern fühlten.

40 Vielleicht verschrieben "Caninisch», nach Canini?

${ }^{41}$ Lichtenbergs Luftpumpe von Nairne, heute noch im Physikalischen Institut Göttingen vorhanden; Abbildung s. Ausstellungskatalog zum 175. Todestag, Städt. Museum Göttingen 1974, S. 56.

42 In der Handschrift astronomisches Zeichen für Sonne = Sonntag.

${ }^{43}$ In der Handschrift astronomisches Zeichen für Mars = Dienstag.

\section{Summary}

Johann Georg Tralles (1763-1822) was installed as professor of natural science at the Bernese Academy in 1785. He introduced there the knowledge and understanding of modern natural science and created an up to date collection of apparatus. These statements make use of manuscript sources of the Bernese State Archives. They are supplemented by two letters by Tralles hitherto unpublished which are interesting documents of the period of enlightenment. We recognize many of the trends of this period, and the author appears as a true pupil of his venerated teacher Lichtenberg.

Dr. phil. Wolfgang Gresky

Am Weißen Stein 11

D-34. Göttingen 